\title{
Responsivität und strategische Adaption im Parteienwettbewerb in den deutschen Bundesländern
}

\author{
Thomas Bräuninger
}

Der Beitrag untersucht am Beispiel der deutschen Landesparteien die Dynamik der programmatischen Entwicklung von Parteien. Es werden zwei Hypothesen abgeleitet und empirisch getestet. Nach der Responsivitätshypothese sollten Parteien auf eine Veränderung der Wählernachfrage nach Politik reagieren, so dass beispielsweise eine Verschiebung der Verteilung der Wählerpräferenzen nach links mit einer Linksverschiebung der Parteipositionen einher geht. Nach der Adaptionshypothese ist zu erwarten, dass der Parteienwettbewerb in einem Mehrparteiensystem bei nicht-vollständiger Information Parteien dazu zwingt, auf die programmatische Positionierung ihrer Mitbewerber strategisch zu reagieren. Datengrundlage sind die Wahlprogramme von Landesparteien in elf Bundesländern seit 1990 sowie Politikbarometer-Umfragen. Die Ergebnisse zeigen erstens, dass ein responsiver Zusammenhang zwischen Wählernachfrage und Parteipositionierung besteht. Zweitens gibt es Hinweise darauf, dass Parteien eine sogenannte Predator-Strategie anwenden, also versuchen, sich dem Sieger der letzten Wahl programmatisch anzunähern.

\section{Einleitung}

Moderne Demokratien sind ohne politische Parteien kaum denkbar. Parteien bzw. ihre Mitglieder repräsentieren und aggregieren gesellschaftliche Interessen, sie streben danach, politische Ämter zu besetzen und dort Einfluss auf politische Entscheidungsprozesse zu nehmen. Mit dieser Charakterisierung unterscheiden sich Parteien von Interessengruppen oder Bürgerinitiativen; sie sind nicht nur ein $\mathrm{Zu}$ sammenschluss von Individuen mit ähnlichen politischen Vorstellungen, die, wie etwa bei Interessengruppen, qua Lobbyarbeit in politischen Entscheidungsprozessen durchgesetzt werden sollen. Parteien sind auch Organisationen, die danach streben, ihre Mitglieder über allgemeine Wahlen in politische Ämter zu bringen. Das heißt, Parteien aggregieren die (inner-)parteilichen Interessen ihrer Mitglieder und sie streben danach, die (außer-)parteilichen Interessen von Wählern repräsentieren zu "dürfen". Mit anderen Worten, bei modernen Parteien ist die Dualität und mitunter das Spannungsverhältnis von Innen- und Außenausrichtung besonders ausgeprägt. Sie sind zumindest stärker als bei anderen politischen Gruppierungen wie Verbänden oder bloßen Wahlbündnissen. 
Dass die beiden impliziten Zielfunktionen einer Partei, Vertretung innerparteilicher Interessen und Erhalt von Wählerstimmen von außerhalb der Gruppe der Parteimitglieder miteinander in Konflikt stehen können, dafür gibt es freilich eine Vielzahl von Beispielen. So wird beispielsweise das von Bundeskanzler Gerhard Schröder im März 2003 präsentierte Reformpaket „Agenda 2010" vor allem als Strategiewechsel der Sozialdemokratischen Partei (SPD) mit dem Ziel, sich Wählerstimmen in der Mitte des parteipolitischen Spektrums zu sichern, interpretiert; ein Strategiewechsel jedoch, der bekanntlich auf erheblichen Widerstand an der Parteibasis stieß (Egle \& Zohlnhöfer 2007: 519). Das muss nicht auf alle Parteien und zu allen Zeiten gleichermaßen zutreffen. Für ideologisch extreme Parteien sollte die Außenperspektive eine geringere Rolle spielen. Mit der oftmals diagnostizierten Abnahme der Parteiidentifikation bzw. geringeren Bedeutung der Parteiidentifikation für das Wahlverhalten sollte der Außenperspektive hingegen eine größere Bedeutung zukommen.

In der vergleichenden Parteienforschung sind beide Sichtweisen, aber je nach Forschungstradition in unterschiedlicher Gewichtung präsent. Klassische Studien zur Genese (Lipset \& Rokkan 1967) und Typologie von Parteiensystemen in modernen Demokratien betonen historische Entwicklungslinien, sozialstrukturelle Basis, Wählerbindung und ideologische Stabilität von Parteien, etwa wenn zwischen agrarischen, konservativen, liberalen und sozialistischen Parteien unterschieden wird (Mair \& Mudde 1998). Studien in der ökonomischen Forschungstradition betonen hingegen stärker die Außenperspektive, also das Streben der Parteien nach Wählerstimmen und Macht. In dieser, zumeist Downs zugeschriebenen Sichtweise erscheint die ideologische oder programmatische Ausrichtung rein instrumentell und auf den Erhalt von Wählerstimmen ausgerichtet (Downs 1957).

Dass in der Regel beide Faktoren von Bedeutung sind, darauf weisen nicht nur Fallstudien zu einzelnen Parteien und ihrem Wandel hin. Studien zum Wählerverhalten zeigen an, dass individuelle Wahlentscheidungen sowohl von der sachpolitischen Beurteilung der Parteien als auch einer (sozialisationsbedingten) Parteiidentifikation abhängen, was umgekehrt für stimmeninteressierte Parteien bedeutet, sich sowohl um die affektive Bindung als auch die sachpolitische Nähe zu ihren Wählern bemühen zu müssen (Pappi \& Shikano 2007). Und schließlich lässt auch die Dynamik der programmatischen Entwicklung von Parteien, wie sie im Längsschnitt im Ländervergleich nachgezeichnet werden kann (Budge et al. 2001; Klingemann et al. 2005), die Vermutung zu, Parteien konkurrierten mit einem sachpolitischen Angebot um Wählerstimmen; wenngleich selten mit dem schlichten Ergebnis des Downschen Basismodells, der Konvergenz am Medianwähler.

Der folgende Beitrag beschäftigt sich mit diesem Themenkreis, indem er die Dynamik der programmatischen Entwicklung deutscher Landesparteien unter die Lupe nimmt. Wie ich in einem ersten Abschnitt zeige werde, weisen die Landesparteien der deutschen Bundesländer durchaus eine erhebliche Veränderung ihrer Positionierung auf der ideologischen Links-Rechts-Dimension auf, so dass sich die Frage stellt, ob dies lediglich einen stochastischen Messfehler oder systematische Determinanten widerspiegelt. Konkret werde ich zum einen untersuchen, inwieweit die empirisch beobachtete Veränderung der Parteipositionen eine Veränderung der 28 
Wählernachfrage reflektiert, also beispielsweise ob mit einer Linksverschiebung der Verteilung der Wählerpräferenzen auch eine Linksverschiebung von Parteipositionen einher geht. Zum anderen sollen die Effekte des Parteienwettbewerbs selbst untersucht werden. Solange die Parteien keine hinreichende Information über die Wählerverteilung haben oder Gleichgewichtpositionen nicht vorhanden sind, kann erwartet werden, dass Parteipositionen sich von Wahl zu Wahl verändern, eben als Reaktion auf den Parteienwettbewerb und nicht aufgrund einer Veränderung von Wählerpräferenzen.

Datengrundlage sind die Wahlprogramme von Landesparteien in elf Bundesländern seit 1990, deren Verortung auf der ideologischen Links-Rechts-Dimension mithilfe des Wordscore-Verfahrens geschätzt werden (Bräuninger \& Debus 2009). Die Dynamik der Wählernachfrage in den einzelnen Bundesländern wird mit Daten der monatlichen Politbarometer-Befragungen abgeschätzt. Die Ergebnisse zeigen, dass durchaus ein Zusammenhang zwischen Wählernachfrage und Parteipositionierung besteht. Parteien scheinen auf eine Verschiebung der Wählerverteilung zu reagieren und zwar insbesondere dann, wenn sich die Wählerverteilung vom eigenen politischen Lager abwendet. Zweitens gibt es Hinweise darauf, dass Parteien eine sogenannte Predator-Strategie anwenden, also versuchen, sich dem Sieger der letzten Wahl programmatisch anzunähern. Dies ist aber freilich noch keine Widerlegung des Parteienwettbewerbs-Arguments. Wie ich im nächsten Abschnitt argumentieren werde, gibt es ohne weitere restriktive Annahmen keine eindeutige optimale Strategie für Parteien. Die Predator-Strategie ist ein plausible, mitnichten aber die einzig mögliche Erklärung für die Dynamik des programmatischen Parteienwettbewerbs. Drittens kann festgestellt werden, dass alle diese Effekte von substantieller Größe sind. Zwar ändern Parteien ihre Programmatik zwischen zwei Wahlen nie radikal, auch „leap-frogging“ (Budge 1994) ist kaum zu beobachten. Dies liegt auch darin begründet, dass die Wählerverteilung weitgehend stabil bleibt. Insofern sich das Elektorat jedoch in eine Richtung bewegt, folgt das Parteiensystem dieser Bewegung. Und schließlich ist festzustellen, dass die obigen Resultate in Einklang mit den Befunden von Budge (1994) und Adams et al. (2004) stehen, die in ländervergleichenden Analysen europäischer Parteien zu ganz ähnlichen Ergebnissen kommen.

Der Beitrag ist wie folgt aufgebaut: Der nächste Abschnitt stellt kursorisch die Literatur zum Parteienwettbewerb vor. In den beiden nachfolgenden Abschnitten erläutere ich die hier verwendeten Maße für die programmatische Ausrichtung der Parteien sowie der Wählernachfrage. Anschließend stelle ich das Analysedesign vor bevor in einem weiteren Abschnitt die Ergebnisse der statistischen Analyse berichtet und diskutiert werden. Ein weiterer Abschnitt fasst die Ergebnisse vor dem Hintergrund der Literatur zum Parteienwettbewerb und der Repräsentation zusammen. 


\section{Die programmatische Ausrichtung von modernen Parteien}

Trotz der umfangreichen Literatur zur Logik des programmatischen Parteienwettbewerbs im Nachgang zu Downs (für einen Überblick siehe Grofman 2004) und zur vergleichenden Deskription und Analyse der programmatischen Ausrichtung von Parteien (Budge et al. 2001), bietet die vergleichende Parteienforschung erstaunlich wenig systematische Erkenntnisse über die Determinanten der programmatischen Entwicklung von Parteien, die über die fallbezogene Nachzeichnung der Entwicklung einzelner Parteien oder Parteifamilien hinausreicht.

Dies mag zum einen daran liegen, dass die Menge potentieller, plausibler Einflussfaktoren groß ist und von institutionellen Faktoren wie dem Wahlsystem, und Regeln der Regierungsbildung bis zu politisch-kulturellen Faktoren, wie der Anzahl an Parteien, dem Mitgliedertypus einer Partei und mithin den Zielen und Präferenzen der Parteimitglieder reicht (Cox 1990). Zum anderen aber lässt bereits der theoretische Befund der generischen Instabilität von Mehrheitsentscheidungen nicht erwarten, dass außer unter sehr restriktiven Bedingungen eindeutige Vorhersagen über die Dynamik des Parteienwettbewerbs gemacht werden können.

Derart restriktive Bedingungen stellen die Annahmen des Downschen Grundmodells dar (Downs 1957): Zweiparteiensystem, eindimensionaler ideologischer oder sachpolitischer Themenraum, ausschließlich ämtermotivierte Parteien, ausschließlich policy-motivierte Wähler, vollständige Information der Parteien über die Wählerverteilung. Bekanntermaßen ergibt sich unter diesen Bedingungen eine eindeutige Gleichgewichtslösung am Median der Wählerverteilung bzw. im Falle einer probabilistischen Wählerentscheidung bei quadratischen Nutzenfunktionen am Mittelwert der Verteilung (Enelow \& Hinich 1984; Lin et al. 1999). Das heißt, für die beiden Parteien ist der Median bzw. Mittelwert die im Hinblick auf das Ziel der Stimmenmaximierung ,beste" programmatische Positionierung. Unter diesen, aber eigentlich nur unter diesen Bedingungen, ergibt sich somit eine eindeutige Erwartung bezüglich der programmatischen Ausrichtung von Parteien bzw. einer Veränderung der Positionierung als Folge einer Verschiebung der Wählerverteilung.

Sind eine oder mehrere der obigen Bedingungen nicht mehr gegeben, dann ist im Allgemeinen nicht mehr von der Existenz einer Gleichgewichtslösung auszugehen und entsprechend können wir auch keine entsprechende Hypothese über die Positionierung der Parteien ableiten. Zwar lassen sich wiederum in bestimmten Situationen Gleichgewichte finden, etwa wenn Kandidaten selbst über ideologische Präferenzen verfügen (Wittman 1977; Calvert 1985; Duggan \& Fey 2005; Smirnov \& Fowler 2007) oder für Wähler non-policy Faktoren eine Rolle spielen (Adams 2005; Groseclose 2001). Doch auch diese Bedingungen sind restriktiv und in vergleichenden empirischen Untersuchungen realer Parteiensysteme schwer überprüfbar. Kurz, es verwundert nicht, dass wir - verglichen mit der Größe des theoretischen Fundus wenig über die Dynamik des programmatischen Parteienwettbewerbs wissen.

Ein weiterer Grund für die Forschungslücke liegt freilich im Mangel an verfügbaren Daten begründet. Zur Überprüfung allein des einfachen Downschen Basismodells bedarf es Informationen über die Art und Veränderung der Wählerverteilung 
respektive des Medianwählers auf einer (zunächst unterstellten) Hauptkonfliktlinie, der Positionierung von Parteien auf dieser Dimension sowie deren Veränderung über die Zeit. Solche Informationen sind für ländervergleichende Studien kaum verfügbar. Die momentan beste Datenbasis hierfür stellt sicherlich das Comparative Manifesto Project (CMP) zur Verfugung. Im Rahmen des CMP-Projekts wurden bis heute über 1500 Wahlprogramme von politischen Parteien in über 30 westlichen Demokratien im Zeitraum ab 1945 inhaltsanalytisch bearbeitet und codiert, so dass die Veränderung der Parteipositionierungen auf wichtigen Politikdimensionen im Zeitverlauf nachvollzogen werden kann (Budge et al. 2001). Die Daten bilden entsprechend die Grundlage für die wenigen empirischen theoriegeleiteten Untersuchungen zum programmatischen Parteienwettbewerb. Budge (1994) geht von Parteiensystemen ohne Gleichgewichtslösung aus und macht weiterhin die plausible Annahme, dass die Parteien die Wählerverteilung nicht kennen bzw. die Wählerverteilung keine sicheren Rückschlüsse über das Wahlverhalten respektive erzielbare Stimmengewinne zulässt. In einer solchen Situation können Parteien nur „Daumenregeln" anwenden, also etwa eine Weiterentwicklung ihrer Programmatik davon abhängig machen, ob ein Richtungswechsel bei der vorgehenden Wahl zu Stimmengewinnen oder -verlusten geführt hat. Budge schließt aus seiner empirischen Untersuchung von etwa 20 Parteiensystemen, dass die Parteien unterschiedliche Strategien für den programmatischen Wandel anwenden, aber auch, dass die programmatischen Positionen weitgehend stabil bleiben.

In Erweiterung dieser Analyse untersuchen Adams et al. (2004) mit Hilfe von Eurobarometer-Daten, ob - entsprechend einer der Kernaussagen der räumlichen Modelle des Parteienwettbewerbs - Veränderungen in der Wählerverteilung beziehungsweise Wählernachfrage nach Politik programmatische Veränderungen auf Seiten der Parteien nach sich ziehen. Die Antwort darauf ist ein Ja, wenngleich sich zeigt, dass nur für solche Parteien eine Adaption belegt werden kann, die von der Verschiebung der Wählerverteilung eindeutig benachteiligt werden, also etwa wenn eine sozialistische Partei mit einem Rechtsruck in der Wählerschaft konfrontiert wird. Das erscheint insofern auch plausibel, als die Parteien wohl kaum vollständige Information über die genaue Verschiebung der Wählerverteilung oder das Entscheidungskalkül aller Wähler besitzen, welche ihnen erlauben würde, einen möglicherweise optimalen programmatischen Wandel zu vollziehen. Wenn Parteien nur die Richtung der Veränderung der Wählerverteilung kennen (aber nicht die genaue Lage der Verteilung), dann ist für benachteiligte Parteien klar, dass sie Wählerstimmen nur dann erhalten, wenn sie die Veränderung im Elektorat nachvollziehen, das heißt sich in die gleiche Richtung bewegen müssen. Bevorzugte Parteien, also linke Parteien, die mit einem Linksruck des Elektorats, oder rechte Parteien, die mit einen Rechtsruck konfrontiert werden, stehen hingegen vor einem, vergleichsweise komfortablen Dilemma. Die Veränderung des Wählerverteilung kann bedeuten, dass sie besser als zuvor positioniert sind - was einen unveränderten programmatischen Kurs implizieren würde -, sie kann aber auch bedeuten, dass sie von der Wählernachfrage „,uberholt" werden - worauf eine Radikalisierung der Programmatik die angemessene Antwort wäre. 
Die Frage, ob und wenn, wie Parteien auf eine veränderte Wählernachfrage nach Politik reagieren, soll im Folgenden für die Landesparteien in den deutschen Bundesländern seit 1990 untersucht werden. Bevor ich mit der empirischen Analyse fortfahre, will ich abschließend nochmals verdeutlichen, warum beobachteter programmatischer Wandel von Parteien nicht notwendigerweise von einer Veränderung der Wählernachfrage herrühren muss - selbst dann, wenn wir von weiteren Faktoren, wie dem Einfluss von non-policy Faktoren auf das Wählerverhalten und damit den Parteienwettbewerb absehen. Grund hierfür ist die eingangs diskutierte generische Instabilität des Parteienwettbewerbs. Abbildung 1 verdeutlicht das Argument.

Betrachten wir zunächst den einfachen Fall, dass ein gegebenes Parteiensystem bei beliebiger, aber hinreichend allgemeiner Wählerverteilung ein Gleichgewicht darstellt. Ein Beispiel hierfür wäre ein Zweiparteiensystem bei eindimensionalem Themenraum. Zu erwarten wäre hier eine Positionierung beider Parteien am Medianwähler (oder dem Mean voter für eine probabilistische Entscheidungsfunktion der Wähler). Bleibt die Wählerverteilung zwischen zwei Wahlen konstant, wäre keine programmatische Veränderung der Parteien zu erwarten. Verändert sich die Wählerverteilung, würden wir von einer entsprechenden Verschiebung der Parteipositionen ausgehen.

Anders sind die Verhältnisse, wenn bei gegebenem Parteiensystem (beispielsweise drei Parteien bei eindimensionalem Themenraum) keine Gleichgewichtspositionen vorhanden sind. In diesem Fall müssen wir auch bei konstanter Wählernachfrage mit programmatischen Veränderungen der Parteien zwischen zwei Zeitpunkten rechnen. Parteien werden versuchen, den programmatischen Wandel ihrer Mitbewerber zu antizipieren und - unter der Zielvorgabe der Stimmenmaximierung entsprechend zu reagieren. Da keine Gleichgewichte existieren, kann eine solche Reaktion nur optimal in der aktuell gegebenen Situation sein; fortwährende Parteibewegungen sind hier nur Reaktionen auf die Dynamik eines Parteienwettbewerbs in einem System ohne optimale ideologische Positionierungen. Empirisch wird wohl der vierte Fall von größter Relevanz sein: die Beobachtung von Parteienbewegungen in Systemen ohne Gleichgewicht bei veränderter Wählernachfrage.

Abbildung 1: Programmatischer Wandel in Abhängigkeit von Wählernachfrage und Parteiensystem

\begin{tabular}{l|c|c|}
\multicolumn{1}{c}{$\begin{array}{c}\text { Parteiensystem mit (multiplem) } \\
\text { Gleichgewicht }\end{array}$} & \multicolumn{1}{c}{$\begin{array}{c}\text { Parteiensystem ohne } \\
\text { Gleichgewicht }\end{array}$} \\
\cline { 2 - 3 } $\begin{array}{l}\text { konstante } \\
\text { Wählernachfrage }\end{array}$ & $\begin{array}{c}\text { Parteienbewegung als } \\
\text { Reine Bewegung } \\
\text { Reaktion auf Parteien- } \\
\text { systemdynamik }\end{array}$ \\
\cline { 2 - 3 } $\begin{array}{l}\text { dynamische } \\
\text { Wählernachfrage }\end{array}$ & $\begin{array}{c}\text { Parteienbewegung als Reaktion } \\
\text { auf veränderte Wähler- } \\
\text { nachfrage }\end{array}$ & $\begin{array}{c}\text { Reaktion auf veränderte } \\
\text { Wählernachfrage und } \\
\text { Parteiensystemdynamik }\end{array}$ \\
\hline
\end{tabular}


In der nachfolgenden Analyse der programmatischen Veränderungen in den Parteiensystemen der Bundesländer sollen deshalb beide Mechanismen betrachtet und untersucht werden; zum einen die Reaktion der Landesparteien auf Veränderungen der Wählernachfrage in dem jeweiligen Bundesland, zum anderen die Reaktion auf die Dynamik des Parteiensystems. Die erste, Responsivitätshypothese unterstellt in Anlehnung an Adams et al. (2004: 593), dass Parteien (zumindest) dann auf eine Veränderung der Wählernachfrage reagieren, wenn sie von der Veränderung negativ betroffenen sind, also wenn Parteien aus dem linken (rechten) Lager mit einer Rechts-(Links-)Verschiebung der Wählerpräferenzen konfrontiert werden.

Hypothese 1: Parteien passen ihre programmatische Ausrichtung einer veränderten Wählernachfrage an, wenn sie von der Veränderung der Wählernachfrage negativ betroffen sind.

Für den zweiten Mechanismus ist die Herleitung einer Hypothese ungleich schwieriger, weil das Fehlen eines Gleichgewichts ja gerade bedeutet, dass es keine Handlungsanweisung gibt, die mit Sicherheit stimmenmaximierend wäre. Welche Strategie wird ein politischer Akteur in dieser Situation einschlagen? Eine mögliche Strategie ist die Orientierung am Gewinner der letzten Wahl; schließlich war dieser mit seinem programmatischen Profil erfolgreich im Wettbewerb um Wählerstimmen. Dies entspricht der Predator-Strategie bei Laver (2005), der in einer Simulationsstudie mögliche Strategien von Parteien zur Stimmenmaximierung in einem Standardmodell eines Mehrparteiensystems bei zwei relevanten Themendimensionen (und damit mit fehlendem Gleichgewicht) untersucht. Nach der PredatorStrategie versuchen Parteien ihren Stimmeanteil dadurch zu erhöhen, dass sie sich der programmatischen Position des Gewinners der vergangenen Wahl annähern. Entsprechend lautet die zweite, Adaptionshypothese:

Hypothese 2: Parteien passen ihre programmatische Ausrichtung der Programmatik des Gewinners der letzten Wahl an.

\section{Dynamik der Parteienpositionierung in den deutschen Bundesländern}

Zur Überprüfung der beiden Hypothesen benötigen wir Information zu den programmatischen Positionen der Landesparteien zu mehreren Zeitpunkten sowie Informationen zur jeweiligen Nachfrage der Wähler nach einer bestimmten Politik. Für die Bestimmung von policy-Positionen politischer Akteure stehen mittlerweile eine Reihe von standardisierten Verfahren zur Verfügung, allerdings sind nur wenige für eine retrospektive Erfassung von Positionen im Längsschnitt geeignet. Im Folgenden verwende ich Präferenzinformationen, die aus einer Analyse der Wahlprogramme von Landesparteien in den elf Ländern der alten Bundesrepublik 
im Zeitraum von 1990 bis 2005 generiert wurden. ${ }^{1}$ Damit sind die Ergebnisse der Analyse zwar nicht unmittelbar in den international-vergleichenden Kontext generalisierbar, das Studium subnationaler Parteiensysteme erlaubt aber, eine Reihe von Kontext- oder intervenierenden Variablen annähernd konstant halten zu können, die sich im internationalen Vergleich als wichtig erweisen, wie beispielsweise das Wahlsystem. Die Beschränkung auf die westdeutschen Bundesländer und den Zeitraum bis 2005 ergibt sich aus der eingeschränkten Verfügbarkeit von Informationen zur Wählernachfrage in den Politikbarometer-Studien. Weiterhin werden nur die Positionierungsstrategien der vier großen Parteien CDU bzw. CSU, SPD, FDP und Bündnis 90/Die Grünen untersucht; kleinere Parteien sind nicht kontinuierlich in mehreren Landtagen vertreten gewesen.

Zur Bestimmung der in Wahlprogrammen formulierten programmatischen Positionen wird auf das Wordscore-Verfahren (Laver et al. 2003) zurückgegriffen. Es geht von der Überlegung aus, dass die Wortwahl politischer Akteure in ihren programmatischen Dokumenten oder Reden nicht nach dem Zufallsprinzip erfolgt. Vielmehr senden beispielsweise Parteien in ihren Wahlprogrammen "ideologische Signale" (Pappi \& Shikano 2004) um ihre Anhängerschaft vor der Wahl zu mobilisieren. Daher gehen Laver et al. (2003) von der Annahme aus, dass man allein aufgrund der relativen Worthäufigkeit eines Dokuments auf dessen programmatische Position oder auch die jeweilige Bedeutung eines Politikfeldes schließen kann. Das Grundprinzip von Wordscore ist daher, die relative Worthäufigkeit eines Textes, dessen Position bzw. Salienz man kennt (so genannte „Referenztexte“), mit der relativen Häufigkeit jedes Wortes eines unbekannten Textes zu vergleichen. Da die Position bzw. Salienz des Referenztexts bekannt ist, kann auf die Position bzw. Salienz des neuen Textes geschlossen werden. Als Referenzwerte können beispielsweise Ergebnisse von Expertenbefragungen dienen (vgl. Benoit \& Laver 2006).

Eine große Bedeutung kommt damit der Auswahl der Referenztexte zu. Diese sollten erstens über eine ähnliche Wortstruktur wie die unbekannten Texte verfügen, zweitens sich inhaltlich auf die Politikfelder beziehen, die Gegenstand der Analyse sind, und drittens möglichst umfangreich sein, um den Anteil informationstragender Wörter zu erhöhen (vgl. Laver et al. 2003: 314f.). Diese Kriterien werden in der Regel von den Wahlprogrammen der Parteien erfüllt. Um die Positionen der deutschen Parteien auf der Landesebene zu bestimmen, werden als Referenztexte die Bundestagswahlprogramme von CDU/CSU, SPD, FDP, Bündnis 90/Die Grünen und der PDS von 1990 und 2002 verwendet.

Die Referenzpositionen zu der hier berücksichtigten Politikdimension - der sozioökonomischen Links-Rechts-Dimension - werden den Expertenbefragungen von Laver \& Hunt (1992) sowie Benoit \& Laver (2006) entnommen. Die Positionen aus der 1989 durchgeführten Befragung von Laver und Hunt werden den Wahlprogrammen der Parteien zur Bundestagswahl 1990 zugewiesen, die Positionen der 2002er Befragung von Benoit und Laver den Bundestagswahlprogrammen von

1 Quelle: Political Documents Archive (http:llwww.polidoc.net), vgl. Bräuninger \& Debus (2008) und Benoit et al. (2009). 
2002. Mit der Berücksichtigung von Referenztexten zu Beginn und gegen Ende der Beobachtungsperiode soll sichergestellt werden, dass Themen und politische Schlagwörter, die im Zeitverlauf aufkommen, verschwinden oder aber ihre Bedeutung verändern, gleichermaßen in die Analyse eingehen. ${ }^{2}$

Der Beobachtungszeitraum umfasst alle Wahlen zwischen der Landtagswahl im Saarland am 28. Januar 1990 und den Wahlen in Baden-Württemberg und Rheinland-Pfalz am 26. März 2006. Da Wahlprogramme einige Monate vor einer Wahl verfasst werden, wird für die Programmformulierung von einem Zeitpunkt von 120 Tagen vor einer Landtagswahl ausgegangen, in den letzten beiden Fällen also dem 26. November 2005. Somit liegen der Analyse die Wahlprogramme von vier Parteien zu insgesamt 44 Landtagswahlen zugrunde.

Abbildung 1 zeigt die Dynamik der Positionierung der Landesparteien auf der sozio-ökonomischen Dimension (1=links bis 11=rechts) im Zeitverlauf. Zumindest drei Dinge sind bemerkenswert. Erstens erhalten wir die erwartete relative Verortung der Parteien auf der sozio-ökonomischen Dimension, nämlich Grüne und SPD im linken Spektrum, CDU/CSU und FDP im rechten Spektrum. Dies gilt für alle Bundesländer und mit nur wenigen Ausnahmen den gesamten Zeitraum. Zweitens gibt es Unterschiede zwischen den Ländern. Die Landesparteien in Berlin oder Nordrhein-Westfahlen scheinen generell eine linkere Position einzunehmen als die Parteien in Hessen oder Baden-Württemberg. Und drittens ist ein Trend des linken Parteienlagers nach rechts zu beobachten. Dies gilt immer für die Grünen und in der Mehrzahl der Länder auch für die SPD. Demgegenüber lässt sich allerdings kein allgemeiner Trend für die FDP oder die CDU/CSU ausmachen. Für die nachfolgende Analyse genügt uns zunächst die Beobachtung, dass die geschätzten Positionen augenscheinlich plausibel sind und Varianz zwischen Parteien und Bundesländern sowie über die Zeit hinweg aufweisen.

2 Die durch das Wordscore-Verfahren bestimmten Rohwerte der Positionen bzw. Salienzen können nicht unmittelbar mit den entsprechenden Referenzwerten verglichen werden. Laver et al. (2003) schlagen deshalb eine affine Transformation der Rohwerte vor, so dass im Ergebnis Mittelwert und Standardabweichung von geschätzten und Referenzwerten übereinstimmen. Im vorliegenden Fall ist dies unangemessen, da beispielsweise die Streuung der landespolitischen Positionen zwischen 1990 und 2007 nicht notwendig mit der Streuung der bundespolitischen Positionen 1990 und 2005 übereinstimmen muss. Wir verwenden deshalb eine Standardisierung, mit der Mittelwert und Standardabweichung der Bundesparteiprogramme 1990 und 2005 (Referenzwerte) mit denen der (geschätzten) Bundesparteiprogramme 1994 und 1998 übereinstimmen. Um eine bessere Vergleichbarkeit mit den Daten des Eurobarometers zu erhalten, wurden die Skalen abschließend von einer 20er Skala auf den Wertebereich 1 (links) bis 11 (rechts) transformiert. Für eine detaillierte Beschreibung des Analyseverfahrens und der Daten siehe Bräuninger \& Debus $(2008,2009)$. 
Abbildung 1: Positionierung der westdeutschen Landesparteien auf der sozioökonomischen Links-Rechts-Dimension 1990 bis 2005
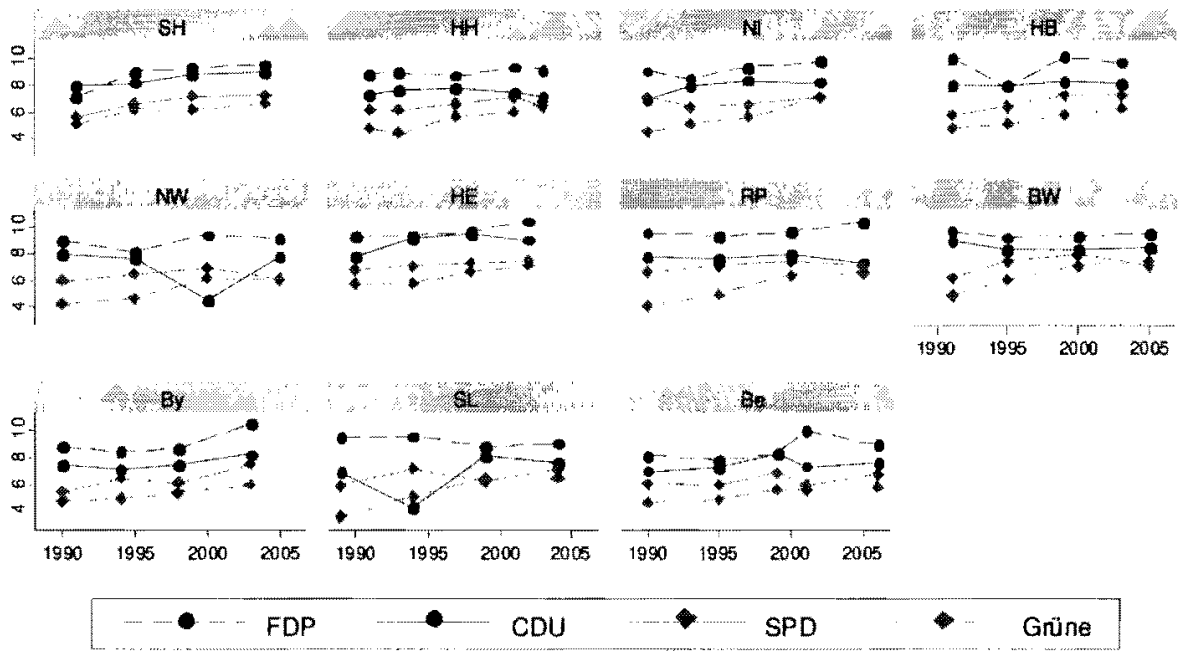

\section{Wählernachfrage}

Zur Abschätzung einer Veränderung der Wählerpräferenzen werden die Selbsteinstufungen von Befragten auf der Links-Rechts-Skala in den Politbarometer-Studien verwendet. Dabei wird den Befragten eine Version der Standardfrage nach der eigenen ideologischen Positionierung vorgelegt, also etwa in den Politbarometern seit 1997 in der folgenden Formulierung:

Wenn von Politik die Rede ist, hört man immer wieder die Begriffe „links" und ,rechts". Wir hätten gerne von Ihnen gewußt, wo Sie sich selbst auf dieser Skala einstufen. Stellen Sie sich dazu bitte noch einmal ein Thermometer vor, das diesmal aber von 0 bis 10 geht. 0 bedeutet sehr links, 10 bedeutet sehr rechts. Mit den Werten dazwischen können Sie Ihre Meinung abgestuft sagen. Wo würden Sie sich einstufen?

Leider stehen nicht für den gesamten Zeitraum, der für unsere Analyse von Interesse ist, Antworten der Befragten auf einer solchen 1 ler-Skala zur Verfügung (Variable v22 des kumulierten Politkbarometer-Datensatzes ZA2391). Vor 1989 wurde die Frage nur in wenigen Jahren und Monaten erhoben und erst seit Januar 1997 kontinuierlich, das heißt monatlich in der obigen Form. Zwischen 1989 und 1996 wurde vielmehr eine einfache 3er-Skala mit den Antwortkategorien ,eher links“", „Mitte/ weder noch" und ,eher rechts" abgefragt, welche dann mit zwei weiteren Fragen 
nach der Rechtsorientierung bzw. Linksorientierung komplettiert wurden (Variablen v23 und v24). Zwischen Januar 1989 und September 1990 konnten die Befragten bei der zweiten Frage lediglich zwischen „etwas links“ und „sehr links“ bzw. „etwas rechts“ und ,sehr rechts“ wählen. Zwischen Oktober 1990 und Dezember 1996 wurde jeweils eine fünfstufige Thermometerskala für eher links bzw. eher rechts orientierte Wähler verwendet, so dass sich in der Kombination mit der ersten Frage hier ebenfalls eine 11-stufige Skala ergibt.

Für die nachfolgenden Analysen werden sämtliche Angaben auf eine Skala im Wertebereich von 1 bis 11 transformiert und als intervallskaliert interpretiert. Für den Zeitraum zwischen Januar 1989 und September 1990 werden den Antwortenkombination „eher links"/"sehr links" bzw. „eher links"/"etwas links" die Werte 1,6 bzw. 3,8 zugeordnet. Befragte mit den Antwortkombinationen „eher rechts", ,etwas rechts" bzw. „eher rechts" $\%$,sehr rechts" erhalten Werte von 8,2 bzw. 10,4 auf der Links-Rechts-Dimension. Nach diesem Umkodierungsschritt werden die LinksRechts-Einstufungen der Befragten aggregiert. Genauer gesagt interpretieren wir die Veränderung des (gewichteten) Mittelwerts der Selbsteinstufungen aller innerhalb eines Kalenderjahres befragter Personen in einem Bundesland als Maß für die Veränderung der Wählerpräferenzen auf $\mathrm{der}$ ideologischen Links-RechtsDimension.

Abbildung 2 zeigt die Veränderung der Wählerpräferenzen nach Bundesländern über die Zeit. Abgetragen sind die Position des Mean voters, das heißt der Mittelwert der Wählerverteilung, sowie die 95-prozentigen Konfidenzintervalle. Die Breite des Konfidenzintervalls reflektiert dabei die Größe eines Bundeslandes. In kleinen Bundesländern wie den Stadtstaaten wurden insgesamt wenige Wähler befragt, so dass hier die Präzision der Bestimmung des Mean voters vergleichsweise gering ausfält. Auch hier zeigt sich, dass sich die Wählerverteilung über die Zeit durchaus ändert und dies nicht in allen Bundesländern in gleicher Weise. Beginnend mit 1989 ist in nahezu allen Ländern ein Trend einer Linksverschiebung des Mean voters zu beobachten. Ein erster Linksruck kann für die erste Hälfte der $90 \mathrm{er}$ Jahre beobachtet werden, 1992 in Hamburg und dem Saarland, etwas später in Hessen und Rheinland-Pfalz. Die Ähnlichkeit der Trends deutet darauf hin, dass hier Determinanten auf der Ebene des Gesamtstaates eine Rolle spielen: die Bewertung der Bundesparteien durch den Befragten oder die Bewertung der makroökonomischen Situation, die eher durch bundesweite denn landesspezifische Indikatoren vermittelt wird (z.B. Arbeitslosigkeitsrate, Inflationsrate). Die ebenfalls bestehenden Unterschiede in den Verläufen zeigen aber, dass landes- oder landespolitikspezifische Faktoren ebenfalls relevant sein können. 
Abbildung 2: Veränderung der Wählerpräferenzen nach Bundesländern (gewichtete Mittelwerte mit 95\%-Konfidenzintervall)

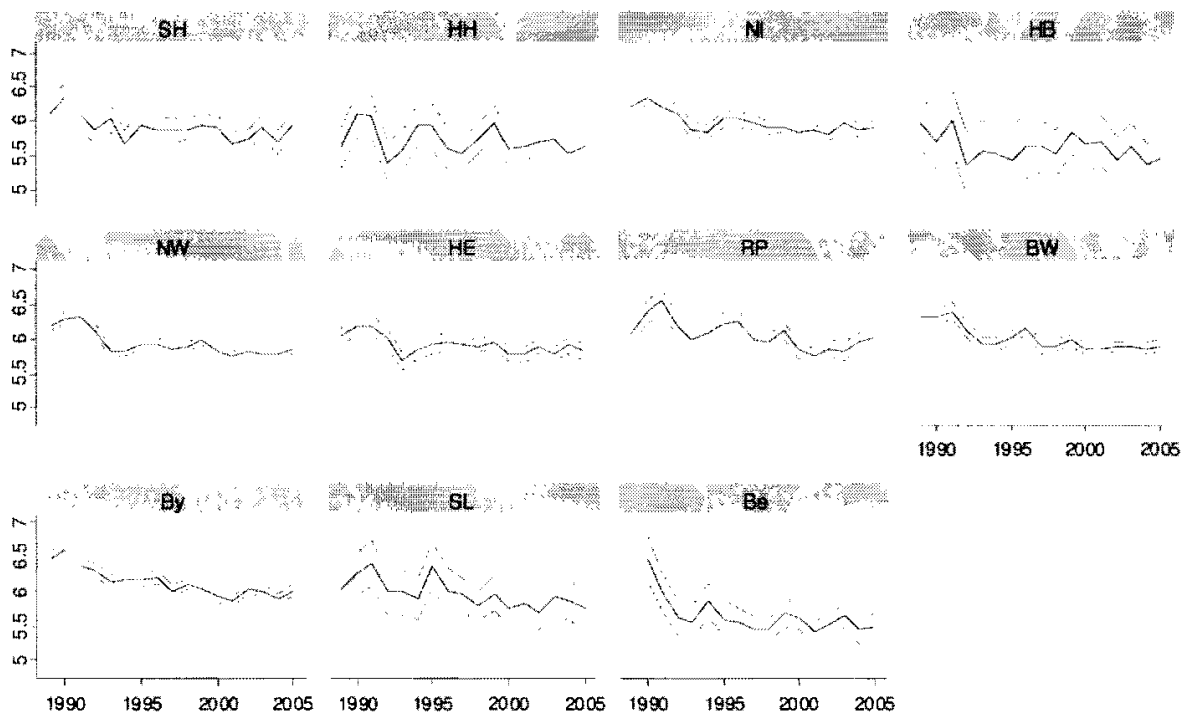

\section{Analysedesign}

Zur Überprüfung der beiden Hypothesen wird ein lineares Regressionsmodell mit einer einfachen, nicht-hierarchischen Mehrebenenstruktur geschätzt:

$$
\begin{array}{ll}
x_{i t}-x_{i, t+1} \sim N\left(\mu+\beta Z_{i t}+\gamma_{[i]}+\delta_{k[i]}, \sigma_{y}^{2}\right), & \text { für } i=1, \ldots, n \\
\gamma_{j[i]} \sim N\left(0, \sigma_{\gamma}^{2}\right), & \text { für } j=1, \ldots, J \\
\delta_{k[i]} \sim N\left(0, \sigma_{\delta}^{2}\right), & \text { für } k=1, \ldots, K .
\end{array}
$$

Dabei steht $j$ für die Partei, $k$ bezeichnet das Bundesland. Im Ergebnis erhält man Schätzungen für den (gemeinsamen) Achsenabschnitt $\mu$ sowie für die Parameter $\gamma$ und $\delta$, welche idiosynkratische Effekte der Parteien beziehungsweise der Bundesländer abbilden.

Die Kovariaten sind im Term $\beta Z_{i t}$ enthalten. Entsprechend dem obigen Argument schätze ich zwei Modelle, zum einen ein Modell, welches ausschließlich die Wählernachfrage als erklärende Variable für die Parteienpositionierung berücksichtigt, zum anderen ein Modell, das die Wählernachfrage und die ideologische Positionierung des Gewinners der letzten Wahl enthält. Für das erste Modell lautet der Kovariaten-Term:

$$
\beta Z_{i t}=\beta_{1}\left(1-D_{i t}^{\text {disad }}\right)\left(x_{i t}^{\text {elec }}-x_{i, t-1}^{\text {elec }}\right)+\beta_{2} D_{i t}^{\text {disad }}\left(x_{i t}^{\text {elec }}-x_{i, t-1}^{\text {elec }}\right),
$$


wobei die Dummy-Variable $D$ anzeigt, ob eine Partei von einer Wählerverschiebung positiv oder negativ betroffen ist, das heißt:

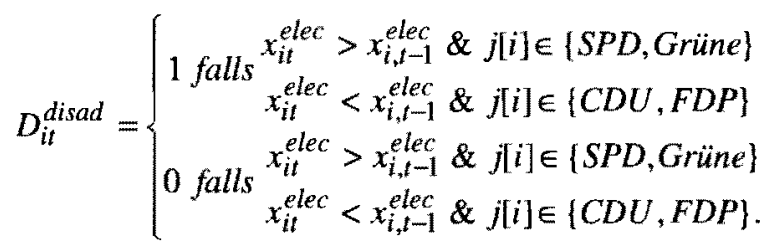

Im zweiten Modell soll zusätzlich der Effekt einer strategischen Adaption an die ideologische Positionierung des Gewinners der letzten Wahl berücksichtigt werden. Ich gehe hier von dem einfachsten denkbaren Fall aus, dass sich alle Parteien gleichermaßen an der Position der Partei mit dem höchsten Stimmenzuwachs orientieren und zwar insbesondere unabhängig davon, welchem ideologischen Lager diese zugehört, wer anschließend in die Regierungsverantwortung kam oder ob der $\mathrm{Zu}$ wachs bei der letzten Wahl letztlich nur einen außerordentlichen Stimmenverlusts bei der vorletzten Wahl ausgleicht (so etwa der fast 20-prozentige Stimmenzuwachs der Hamburger CDU bei der Bürgerschaftswahl 2004).

Bezeichnet $\hat{x}_{k, t-1}$ die Position der Partei mit dem größten Stimmenzuwachs bei einer Wahl in Bundesland $k$ zum Zeitpunkt $t-1$, dann ergibt sich der KovariatenTerm in Modell $2 \mathrm{zu}$ :

$$
\begin{aligned}
\beta Z_{i t} & =\beta_{1}\left(1-D_{i t}^{\text {disad }}\right)\left(x_{i t}^{\text {elec }}-x_{i, t-1}^{\text {elec }}\right)+\beta_{2} D_{i t}^{\text {disad }}\left(x_{i t}^{\text {elec }}-x_{i, t-1}^{\text {elec }}\right) \\
& +\beta_{3}\left(\hat{x}_{k[i], t-1}-x_{i, t-1}\right) .
\end{aligned}
$$

\section{Test der Responsivitäts- und der Adaptionshypothese}

Nach den beiden Hypothesen erwarten wir positive Werte für die drei zu schätzenden Parameter $\beta_{1}, \beta_{2}$ und $\beta_{3}$. Der Koeffizient $\beta_{1}$ gibt an, wie eine Partei auf eine Verschiebung des Mean voters auf der Links-Rechts-Dimension reagiert, wenn dieser sich dem ideologischen Lager der Partei zuneigt, also beispielsweise für die SPD nach links bewegt. Der Koeffizient $\beta_{2}$ zeigt die Responsivität einer Partei an, die auf eine Entfernung des Mean voters reagiert. Da sowohl der Wähler- als auch der Parteienraum auf eine Skala von 1 bis 11 transformiert wurde, entspräche ein Koeffizient von +1 einer Positionsverschiebung der Partei um den selben Betrag und in die selbe Richtung. Wir müssen hier allerdings berücksichtigen, dass wir trotz der vorgenommenen Reskalierung nicht von identischen Skalen ausgehen können. Die Positionen der Parteien werden aus den Wahlprogrammen der Parteien auf der Basis von Referenzwerten geschätzt, die Expertenumfragen zur ideologischen Positionierung der Parteien entstammen. Die Position des Mean voters wird anhand von Selbsteinschätzungen der Wähler in Umfragen bestimmt. Wir müssen davon ausgehen, dass Experten dazu tendieren, bei - insbesondere vergleichenden - Fremdeinschätzungen die Unterschiede von Objekten respektive die Polarisierung eines 
Parteiensystems zu betonen. Wähler, nach ihrer eigenen Positionierung befragt, neigen hingegen dazu, extreme Positionen zu verneinen. Deshalb sind beide Skalen nicht unmittelbar vergleichbar, wir müssen für die Wählerverteilung mit einer geringeren Varianz und Konzentration von Positionen in der Mitte der Skala rechnen. Das ist aber genau das, was wir oben bereits beobachtet haben. Für die strategische Adaption schließlich ist nach Hypothese 2 ebenfalls ein positiver Koeffizient zu erwarten. Befindet sich der Gewinner der letzten Wahl rechts einer Partei, dann erwarten wir, dass diese Partei sich nach rechts, auf die Position des Gewinners zubewegt.

Tabelle 1 sowie Abbildungen 4 und 5 zeigen die Ergebnisse einer Bayesianischen Schätzung der Parameter für die beiden Modellspezifikationen 1 und 2. Eine Bayesianische Schätzung hat im vorliegenden Fall zwei Vorteile. Zum einen erhalten wir als Ergebnis nicht eine einzelne Punktschätzung eines Parameters - wie in der klassischen Inferenzstatistik -, sondern die gesamte, posterior Verteilung aller Parameterwerte, die zu den vorliegenden Daten "passt". Dies ist insbesondere dann von Vorteil, wenn nur wenige Beobachtungen vorliegen, so dass die geringe Datenmenge kaum eine einzelne oder kleine Menge an Parameterkonstellation identifizieren kann (im Sinne einer Maximierung der Likelihood-Funktion im ML-Ansatz). Zudem sind die Konfidenzintervalle der Inferenzstatistik bei Vollerhebungsdaten nicht wirklich sinnvoll interpretierbar. Die posterior Verteilungen der Bayesianischen Schätzung haben hingegen eine natürlich Interpretation: basierend auf der Information der Daten geben sie den Wertebereich an, in dem die Parameter liegen können.

Abbildung 4: Ergebnisse der Parameterschätzung für Modell 1

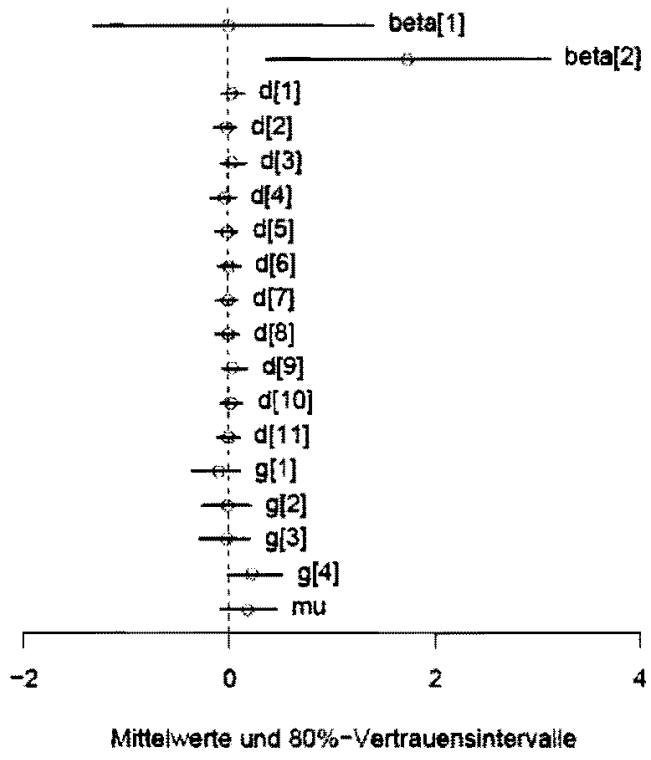


Ein zweiter Vorteil besteht darin, dass das angewandte Verfahren der Markov Chain Monte Carlo-Simulation erlaubt, das Modell trotz fehlender Werte auf der abhängigen Variablen zu schätzen. Fehlende Werte werden als Parameter interpretiert und im Zuge der iterative Bestimmung der posterior Verteilungen mitgeschätzt. Dies erlaubt uns, adäquat mit den Längsschnittcharakter der Daten umzugehen. Wahlprogramme werden nur alle vier bis fünf Jahre verfasst, Daten für Parteipositionen liegen somit mit Vier- oder Fünfjahresspannen vor. Politbarometerumfragen werden monatlich durchgeführt und wurden hier auf Jahresebene aggregiert. Daten zur Wählernachfrage stehen damit für jedes Jahr zur Verfügung. Da wir nach der Responsivitätshypothese davon ausgehen, dass ein Wahlprogramm zum Zeitpunkt der Abfassung (120 Tage vor dem Wahltag) die Veränderung der Wählerverteilung an diesem Zeitpunkt reflektiert, erlauben uns die jährlichen Daten zur Wählernachfrage umgekehrt die Position eines hypothetischen Wahlprogramms zu schätzen, das innerhalb der Wahlperiode verfasst worden wäre. Damit erhalten wir freilich keine zusätzliche Information zur Schätzung der $\beta$-Parameter, aber eine modellgemäße Interpolation der Parteipositionen zwischen zwei Wahlen. Bei der Schätzung des zweiten Modells ist diese Interpolation nicht möglich, da hier auf Seite der unabhängigen Variablen auch die Wahlergebnisse eingehen, die selbst nur alle vier oder funf Jahre vorliegen.

Tabelle 1: Posterior Verteilungen der Modellparameter

\begin{tabular}{llllllll}
\hline & $\begin{array}{c}\text { Mittel- } \\
\text { wert }\end{array}$ & SD & 2.5 & 25 & 50 & 75 & 97.5 \\
\hline $\begin{array}{c}\text { Modell 1 } \\
\text { Positive Veränderung Wäh- } \\
\quad \text { lernachfrage }\left(\beta_{1}\right)\end{array}$ & -0.05 & 1.02 & -2.04 & -0.74 & -0.04 & 0.62 & 1.93 \\
$\quad \begin{array}{l}\text { Negative Veränderung } \\
\quad \text { Wählemachfrage }\left(\beta_{2}\right)\end{array}$ & 1.78 & 1.01 & -0.19 & 1.09 & 1.76 & 2.46 & 3.77 \\
$\begin{array}{c}\text { Modell 2 } \\
\text { Positive Veränderung Wäh- } \\
\quad \text { lernachfrage }\left(\beta_{1}\right)\end{array}$ & 0.48 & 1.13 & -1.83 & -0.25 & 0.47 & 1.22 & 2.67 \\
$\quad \begin{array}{c}\text { Negative Veränderung } \\
\quad \text { Wählernachfrage }\left(\beta_{2}\right)\end{array}$ & 1.51 & 1.18 & -0.76 & 0.72 & 1.49 & 2.32 & 3.88 \\
$\quad \begin{array}{c}\text { Gerichteter Abstand zum } \\
\text { Wahlgewinner } t-1\left(\beta_{3}\right)\end{array}$ & 0.10 & 0.05 & 0.00 & 0.06 & 0.10 & 0.13 & 0.20 \\
\hline
\end{tabular}

Wie Abbildung 4 zeigt, findet die erste, Responsivitätshypothese Bestätigung in den Daten. Es liegen mehr als 80 Prozent der posterior-Verteilung von $\beta_{2}$ (in der Abbildung: beta[2]) im positiven Wertebereich. Damit weisen die vorliegenden Daten darauf hin, dass Landesparteien in der Tat ihre programmatische Ausrichtung einer veränderten Wählernachfrage anpassen, und zwar dann, wenn sie von der Veränderung der Wählernachfrage negativ betroffen sind. Nach Tabelle 1 beträgt der Mittelwert der Parameterverteilung 1.78, was einen substantiellen Effekt ausmacht. Es bedeutet, dass beispielsweise bei einem Rechtsruck des Mean voters um 
0.5 Einheiten auf der Links-Rechts-Dimension das Parteiensystem eine Rechtswendung um 0.89 macht.

Demgegenüber erstreckt sich das Kredibilitätsintervall von $\beta_{1}$ (beta[1]) gleichermaßen über den positiven und den negativen Wertebereich. SPD und Grüne, die mit einer Linksverschiebung der Wählerverteilung konfrontiert werden, bzw. CDU/CSU und FDP, die eine Verschiebung des Mean voters nach rechts beobachten, reagieren nicht bzw. nicht systematisch. Das hatten wir auch erwartet und das Ergebnis steht zudem im Einklang mit den Ergebnissen der international vergleichenden Responsivitätsstudie von Adams et al. (2004). Die übrigen Parameterschätzungen sind hier nicht von sonderlichem Interesse. Wir halten lediglich fest, dass der positive Wert für $\mu(\mathrm{mu})$ einen allgemeinen Trend der Parteipositionierungen nach rechts anzeigt, der im Falle von Bündnis 90/Die Grünen nochmals stärker ausfällt ( $\gamma_{\text {Grüne }}$ bzw. g[4]).

Abbildung 5: Ergebnisse der Parameterschätzung für Modell 2

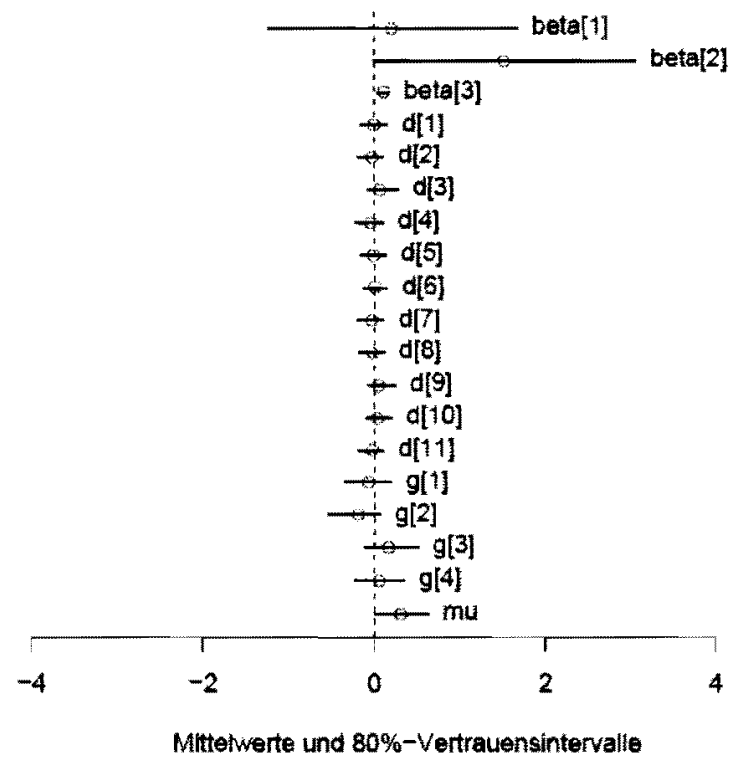

Wenden wir uns der Frage zu, ob wir in den Parteiensystemen der Bundesländer auch Evidenz für die zweite, Adaptionshypothese finden können. Abbildung 5 zeigt die Ergebnisse für Modell 2, indem mit $\beta_{3}$ (beta[3]) ein zusätzlicher Parameter für den Differenzvektor zwischen Parteiposition bei der vorhergehenden Wahl und der Position des Gewinners der Wahl $\left(\hat{x}_{k[i], t-1}-x_{i, t-1}\right)$ geschätzt wird. Auch Hypothese 2 kann als vorläufig bestätigt betrachtet werden. Nach Tabelle 1 beträgt der Mittelwert der posterior-Verteilung 0.0976, wobei das 95-prozentige Kredibilitätsintervall vollständig im positiven Wertebereich liegt. Die „Wahlverlierer“ reagieren also in der nachfolgenden Wahl mit einer Annäherung an die Position des Wahlgewinners und zwar um durchschnittlich 10 Prozent der vorherigen Distanz. Das mag auf den 
ersten Blick keinen großen Sprung bedeuten und mitnichten eine vollständige Übernahme der Programmatik des Gewinners. Das ist aber auch nicht zu erwarten. Erstens impliziert die Verbindung von Partei und ihrer traditionellen Wählerklientel eine nicht zu vernachlässigende Immobilität der Parteien im ideologischen Raum. Zweitens handelt es sich bei der Position des Wahlgewinners in einem Mehrparteiensystem nicht um eine Gleichgewichtsposition entsprechend der des Medians (oder Mean voters) im Zweiparteiensystem. Eine vollständige Übernahme der programmatischen Position des Wahlgewinners wäre im Hinblick auf die Stimmenmaximierung also kaum zielführend. Abschließend ist festzuhalten, dass sich die Ergebnisse für die übrigen Parameter zwischen Modell 1 und 2 nur geringfügig unterscheiden.

\section{Fazit}

Der vorliegende Beitrag untersucht eine der Kernthesen der Theorie des räumlichen Parteienwettbewerbs, nämlich die erwartete Reaktion des Parteiensystems auf eine Veränderung der Wählerpräferenzen. Aus demokratietheoretischer Sicht steht die Nachfrage von Wählern nach Politik am Anfang einer langen Kette von Delegationsbeziehungen (Powell 2000). Seit den grundlegenden Arbeiten von Downs (1957) haben eine Vielzahl von Arbeiten Argumente vorgelegt, wann und wie Kandidaten und Parteien als die zweiten Glieder der Delegationskette auf unterschiedliche Verteilungen von Wählerpräferenzen sowie deren Veränderung reagieren. Empirische Studien haben mittlerweile Evidenz gesammelt, dass Veränderungen in Parteipositionen tatsächlich vorausgegangene Veränderungen in den Positionen der Wähler widerspiegeln (Adams et al. 2004, 2006; Erikson et al. 2002; Ezrow 2007).

Gleichwohl bleiben die Ergebnisse zur policy-Responsivität von Parteien mager, was angesichts der demokratietheoretischen Zentralität des Konzepts bedauerlich ist. Der vorliegende Beitrag beschäftigt sich mit diesem Themenkreis, indem er für die Parteiensysteme der westdeutschen Bundesländer ab 1990 untersucht, ob sich Veränderungen in den Positionierungen der Landesparteien gemäß dem Konzept der Responsivität erklären lassen. Die Ergebnisse zeigen, dass zwei Hypothesen, die sich aus einem Standardmodell des Parteienwettbewerbs ableiten lassen, der Überprufung standhalten: Parteien reagieren auf eine veränderte Wählernachfrage und zwar dann, wenn die Veränderung sie negativ betrifft. Und Parteien orientieren sich an ihren Mitbewerbern; sie adaptieren an die Position des Gewinners der letzten Wahl.

So ermutigend diese Ergebnisse sind, so vielfältig sind die sich daraus ergebenden Fragen. Auf der theoretischen Ebene stellt sich die Frage nach einem geschlossenen Modell der Responsivität, in dem Parteienbewegungen als Reaktion auf veränderte Wählerpräferenzen und die Dynamik des Parteienwettbewerbs verstanden werden können. Hinsichtlich des Parteienwettbewerbs scheinen wir von einer Lösung noch ein gutes Stïck entfernt. Angesichts der Komplexität rationaler Erwägungen im Mehrparteienwettbewerb in mehrdimensionalen Politikräumen gibt es gute Gründe, die „metaphysische Überzeugung, Gleichgewichte seien immer ,ir- 
gendwo' und warteten darauf entdeckt zu werden" (Cederman 1997: 34f.; eigene Übersetzung), aufzugeben und der Untersuchung des Parteienwettbewerbs weniger anspruchsvolle - wenngleich gleichermaßen voraussetzungsvolle - Annahmen über das Akteursverhalten zugrunde zu legen (z.B. Kollman et al. 2003; de Marchi 1999; Laver 2005).

Auf der empirischen Seite ist die in der Literatur gängige Standardkonzeption der policy-Repräsentation auf einer ideologischen Links-Rechts-Dimension wenig zufrieden stellend (z.B. Powell 2000). Zum einen hängt das Wählerverhalten und mit dessen unterstellter Antizipation durch Parteien auch der Parteienwettbewerb in der Regel von mehreren Themendimensionen ab (Holmberg 2000). Zum anderen spielen beim Wählerverhalten eben noch andere, non-policy Faktoren eine bedeutende Rolle (Adams et al. 2005). Auch haben wir im vorliegenden Beitrag die Präferenzen der Wähler stark komprimiert betrachtet. Eingang in die Analyse hat letztlich nur der Mean voter, also der Mittelwert der Wählerverteilung gefunden. Es gibt jedoch gute Gründe anzunehmen, dass auch die Diversität der Wählerpräferenzen ihre Spiegelung in der Diversität der Parteipositionen findet (Ezrow 2007). Und schließlich haben wir den möglichen Einfluss der Bundespolitik schlicht vernachlässigt. Wie auch immer, die Ergebnisse zeigen, dass policy-Responsivität in den Parteiensystemen der Bundesländer vorhanden ist und das Studium der Landespolitik ein lohnendes Feld sein kann.

\section{Literatur}

Adams, James, Clark, Michael, Ezrow, Lawrence \& Glasgow, Garrelt (2004): Understanding Change and Stability in Party Ideologies: Do Parties Respond to Public Opinion or to Past Election Results? British Journal of Political Science 34: 589-610.

Adams, James, Clark, Michael \& Ezrow, Lawrence (2006): Are Niche Parties Fundamentally Different from Mainstream Parties? The Causes and Electoral Consequences of Western European Parties' Policy Shifts, 1976-1998, American Journal of Political Science 50: 513-529.

Adams, James F., Merrill, Samuel \& Grofman, Bernard (2005): A Unified Theory of Party Competition. Cambridge: Cambridge University Press.

Benoit, Kenneth \& Laver, Michael (2006): Party Policy in Modern Democracies. London: Roulledge.

Benoit, Kenneth, Bräuninger, Thomas \& Debus, Marc (2009): Challenges for Estimating Policy Preferences: Building Up an Open Access Resource of Political Documents, German Politics (im Erscheinen).

Bräuninger, Thomas \& Debus, Marc (2008): Der Einfluss von Koalitionsaussagen, programmatischen Standpunkten und der Bundespolitik auf die Regierungsbildung in den deutschen Ländern, Politische Vierteljahresschrift 49: 309-338.

Bräuninger, Thomas \& Debus, Marc (2009): Parteienwettbewerb in den Bundesländern. (in Vorbereitung). 
Budge, Ian (1994): A New Theory of Party Competition: Uncertainty, Ideology and Policy Equilibria Viewed Comparatively and Temporally, British Joumal of Political Science 24: 443-467.

Budge, Ian, Klingemann, Hans-Dieter, Volkens, Andrea, Bara, Judith \& Tanenbaum, Eric (2001): Mapping Policy Preferences: Estimates for Parties, Electors, and Governments 1945-1998. Oxford: Oxford University Press.

Calvert, Randall L. (1985): Robustness of the Multidimensional Voting Model: Platform Motivations, Uncertainty, and Convergence, American Joumal of Political Science 29: $69-95$.

Cederman, Lars-Erik (1997): Emergent Actors in World Politics. Princeton, NJ: Princeton University Press.

Cox, Gary (1990): Centripetal and Centrifugal Incentives in Electoral Systems, American Journal of Political Science 34: 903-935.

de Marchi, Scott (1999): Adaptive Models and Electoral Instability, Joumal of Theoretical Politics 11: 393-419.

Downs, Anthony (1957): An Economic Theory of Democracy. New York: Harper.

Duggan, John, Fey, Mark (2005): Electoral Competition with Policy-motivated Candidates, Games and Economic Behavior 51: 490-522.

Egle, Christoph \& Zohlnhöfer, Reimut (2007): Ende des Rot-grünen Projektes: Eine Bilanz der Regierung Schröder 2002-2005. Wiesbaden: VS Verlag.

Enelow, James, Hinich, Melvin (1984): The Spatial Theory of Voting. Cambridge: Cambridge University Press.

Erikson, Robert, MacKuen, Michael \& Stimson, James (2002): The Macro Polity. Cambridge: Cambridge University Press.

Ezrow, Lawrence (2007): The Variance Matters: How Party Systems Represent the Preferences of Voters, Journal of Politics 69: 182-192.

Grofman, Bernard (2004): Downs and Two-Party Convergence, Annual Review of Political Science 7: $25-46$.

Groseclose, Timothy (2001): A Model of Candidate Location When One Candidate Has a Valence Advantage, American Journal of Political Science 45: 862-886.

Holmberg, Sören (2000): Issue Agreement, in Esaiasson, P. \& Heidar, K. (Hrsg.): Beyond Westminster and Congress: The Nordic Experience. Colombus, OH: Ohio State University Press.

Klingemann, Hans-Dieter, Volkens, Andrea, Bara, Judith, Budge, Ian \& McDonald, Michael (2005): Mapping Policy Preferences 1I: Estimates for Parties, Electors and Governments in Central and Eastern Europe, European Union and OECD 1990-2003. Oxford: Oxford University Press.

Kollman, Ken, Miller, John H. \& Page, Scott E. (2003): Political Institutions and Sorting in a Tiebout Model, in Kollman, Ken \& Miller, John H. (Hrsg.): Computational Models in Political Economy (187-212). Cambridge: MrT Press.

Laver, Michael (2005): Policy and the Dynamics of Political Competition, American Political Science Review 99: 263-281.

Laver, Michael, Benoit, Kenneth \& Garry, John (2003): Extracting Policy Positions from Political Texts Using Words as Data, American Political Science Review 97: 311 - 331. 
Laver, Michael \& Hunt, Ben W. (1992): Policy and Party Competition. New York/London: Routledge.

Lin, Tse-Min, Enelow, James \& Dorussen, Han (1999): Equilibrium in Multicandidate Probabilistic Spatial Voting, Public Choice 98: 59-82.

Lipset, Seymour M. \& Rokkan, Stein (1967): Cleavage Structures, Party Systems and Voter Alignments: An Introduction, in Lipset, Seymour M. \& Rokkan, Stein (Hrsg.): Party Systems and Voter Alignments: Cross-National Perspectives (1-64). New York/London: The Free Press.

Mair, Peter \& Mudde, Cas (1998): The Party Family and its Study, Annual Review of Political Science 1: 211-229.

Pappi, Franz U. \& Shikano, Susumu (2004): Ideologische Signale in den Wahlprogrammen der deutschen Bundestagsparteien 1980 bis 2002. Mannheim: MZES Working Papers Nr. 76.

Pappi, Franz U. \& Shikano, Susumu (2007): Wahl- und Wählerforschung. Forschungsstand Politikwissenschaft. Baden-Baden: Nomos.

Powell, G. Bingham (2000): Elections as Instruments of Democracy: Majoritarian and Proportional Visions. New Haven/London: Yale University Press.

Smimov, Oleg \& Fowler, James H. (2004): Moving with the Mandate: Policy-Motivated Parties in Dynamic Political Competition. Typescript (University of California, Davis).

Smimov, Oleg \& Fowler, James H. (2007): Policy-Motivated Parties in Dynamic Political Competition, Journal of Theoretical Politics 19: 9-31.

Wittman, Donald (1977): Platforms with Policy Preferences: A Dynamic Model, Journal of Economic Theory 14: 180-189. 